\title{
Object-Based Building Extraction from High Resolution Satellite Imagery
}

\author{
R. Attarzadeh ${ }^{\mathrm{a}, *}$, M. Momeni ${ }^{\mathrm{b}}$ \\ a Islamic Azad University, Maybod branch, Maybod, Yazd, Iran- r.attarzadeh@gmail.com \\ ${ }^{\mathrm{b}}$ Department of Surveying Engineering, Faculty of Engineering, University of Isfahan, Hezar Jerib Ave., Isfahan, Iran
}

Commission IV, WG IV/2

KEY WORDS: Extraction, Classification, Detection, Object, Algorithm, High Resolution

\begin{abstract}
:
Automatic building extraction from high resolution satellite imagery is considered as an important field of research in remote sensing and machine vision. Many algorithms for extraction of buildings from satellite images have been presented so far. These algorithms mainly have considered radiometric, geometric, edge detection and shadow criteria approaches to perform the building extraction. In this paper, we propose a novel object based approach for automatic and robust detection and extraction of building in high spatial resolution images. To achieve this goal, we use stable and variable features together. Stable features are derived from inherent characteristics of building phenomenon and variable features are extracted using SEparability and THresholds analysis tool. The proposed method has been applied on a QuickBird imagery of an urban area in Isfahan city and visual validation demonstrates that the proposed method provides promising results.
\end{abstract}

\section{INTRODUCTION}

Automatic building extraction from high resolution satellite imagery is considered as an important field of research in remote sensing and machine vision. So far, many algorithms have been presented for the extraction of buildings from satellite image. These algorithms have mainly considered radiometric, geometric, edge detection and shadow criteria approaches. With the advent of high resolution satellite imagery, a new source has been provided for building extraction algorithms. Such spatial resolution clarifies a large number of details in urban area that have facilitated extraction of urban phenomenon such as roads and buildings.

Since in the high spatial resolution satellite imagery, the pixel size is much smaller than the authentic size of the building, the group of pixels represent a building. Therefore the analysis taken into consideration should be object based image analysis which is more prominent today, rather than pixel-based approach. Object Based Image Analysis allows us to use the other spectral information such as average value of each band, minimum and maximum value, variance along with spatial information such as distance, neighborhood and topology by considering image objects which results from the segmentation as processing unit (Blaschke, 2010).

Here, the human visual system acts on the basis of understanding typical patterns and their relation with real objects. In addition to gray value in this system, such other features as texture, shape, size, and inter-objects relationships are effective in developing these patterns. A similar interpretational approach is used in object based image analysis of remote sensing imagery, though accessing complexity and workmanship of human perception is not possible (Nussbaum et al., 2008).

Due to the numerous limitations in only using spectral features for building extraction, particularly where there is a spectral overlap between building and other urban phenomena, objectbased image analysis seems necessary because of taking spatial, contextual, and geometric concepts into account.

Several studies have been conducted in relation to building extraction from high resolution satellite imagery using object based image analysis. One of the first studies was carried out by Hofmann in 2001. In this study the object based image analysis is used to extract the informal settlement of Cape Town from Ikonos image. Image segmentation and successive classification of image objects have been done by complex class hierarchy using different features such as shape, size, context and texture. The same technique is used in more enhanced and improved way to extract an informal settlement of Rio de Janerio. The method is used in a more simplified class hierarchy in a Quickbird image with fuzzy membership function and their combinations (Hofmann et al., 2008).

In another research a Quickbird image was used for object based image analysis to extract urban features for making a building inventory of Bangkok city (Dutta and Serker, 2005). Multi level segmentation approach was used to detect different urban object in appropriate size and the segments are classified in a hierarchical scheme. Fuzzy membership function is applied to utilize different shape, size and spectral feature characteristics for building detection.

In another study, a VHR orthoimage is used to extract the very dense urban slum dwellings and population living in it is estimated (Aminipouri et al., 2009). Chessboard segmentation is applied first to reduce the area of the interest and then multiresolution segmentation is used for efficient and meaningful segmentation of the image to carry out classification of buildings. Fuzzy membership and nearest neighbour classification are applied for extraction of different types of roof structures based on colour, shape, size, texture and context.

\footnotetext{
* Corresponding author.
} 


\section{BUILDING EXTRACTION APPROACH}

\subsection{Principle}

The presented paper tends to extract building using object based image analysis considering human visual system workmanship.

The proposed algorithm considers a phenomena as a building while observing a satellite image in an object-based approach (not a pixel-based approach) that: 1) has a regular geometric shape, 2) has homogenous building body and a significant variation in transition to close neighbourhood, and 3) is high and consequently has a direct neighbourhood with shadow.

In this paper, those features that have derived from this definition are so called stable features. Stable features in a general algorithm may be implemented on images from different areas and with different sensors as a stable part. In this general algorithm, since buildings shape and construction materials vary even in the same geographical regions, a flexible core is proposed to cover the image under study. Features leading to optimal separation of building class and other classes (two by two) in the image are extracted in this section. This paper classifies these features as variable features. To extract these features, it is essential to use an analysis tool. SEaTH analysis tool presented by Nussbaum has been used in this paper. In this method by using Jeffries-Matusita measure, features are extracted as optimal features in appropriate separation of probability distribution function for the training samples belonging to different classes.

Thereafter, using aforementioned method and choosing image objects belonging to building class and other classes detected in the image, features leading to optimal separation between building class and other classes and the required threshold are determined for reaching such separation.

Algorithm mentioned above is shown in the following figure:

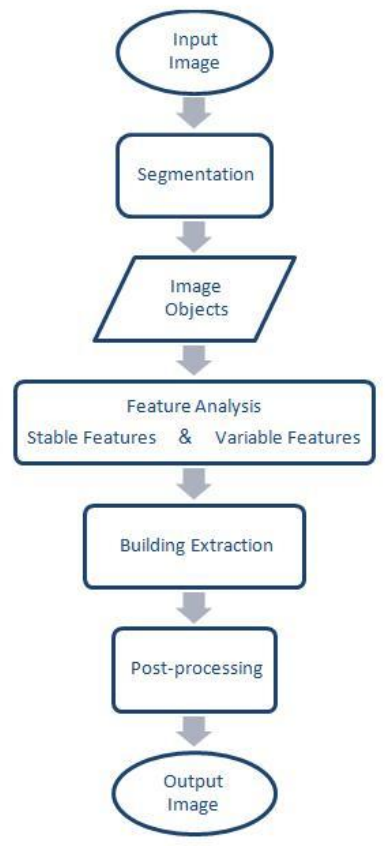

Figure 1. Workflow of the proposed method

\subsection{Data}

The described methodology has been applied on QuickBird multi spectral images of an urban area in Isfahan city (figure 2). As observed, this image includes buildings with different size, roof, shape and arrangement.

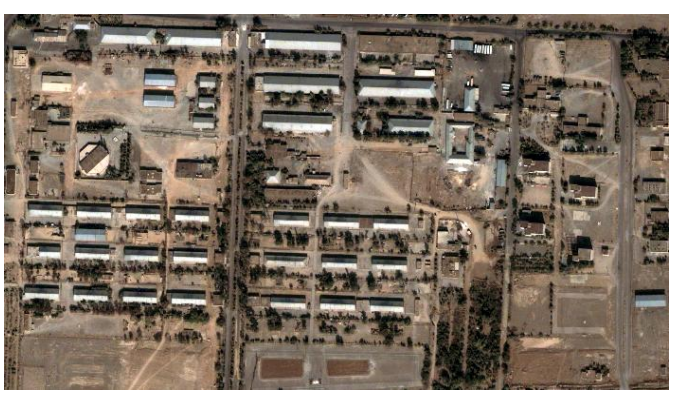

Figure 2. The original image

\subsection{Segmentation}

At the first stage an image to be analysed is segmented into individual image objects in an object based approach (Nussbaum et al., 2008). The image pixels from the image are grouped to form objects in a segmentation process. The created image objects should present the objects in reality. In this research multiresolution segmentation algorithm has been used to create image objects. multi-resolution segmentation is a bottom up region-merging technique starting with one-pixel objects. In numerous subsequent steps, objects are grouped into a larger object based on spectral similarity, contrast with neighbouring objects, and shape characteristics of the resulting object. In each step, that pair of adjacent image objects is merged which results in the smallest growth of the defined heterogeneity. If the smallest growth exceeds the threshold defined by the scale parameter, the process stops (Benz et al., 2004). In this algorithm, the proximity degree of gray value to each other in an image object is determined by Scale Parameter. The bigger this scale parameter value, the smaller this proximity becomes and so the size of the objects will be bigger. In this study, in a trial and error process, in order to obtain optimal results, the value of this parameter has been considered 40 . It is to be noted that choosing appropriate scale parameter prevents over and under segmentation, though accessing ideal segmentation considering this fact that there is numerous image objects with different heterogeneity in a satellite image is not possible. Selected scale parameter considers colour and shape factor simultaneously. Though, in many cases the colour factor is the most effective parameter in the creation process of image objects, considering shape factor leads to quality improvement of the produced objects. In this research, colour factor is weighted with 0.7 and shape factor is weighted with 0.3 . It is mentioned that shape factor is divided into two parameters of smoothness and compactness and both weights are considered 0.5 in this case study (figure 3 and figure 4). 


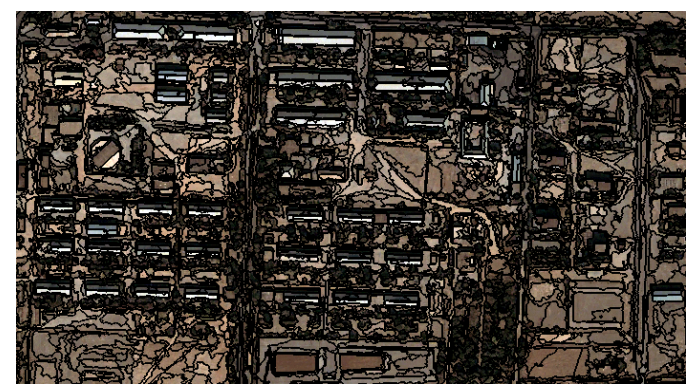

Figure 3. Segmented image

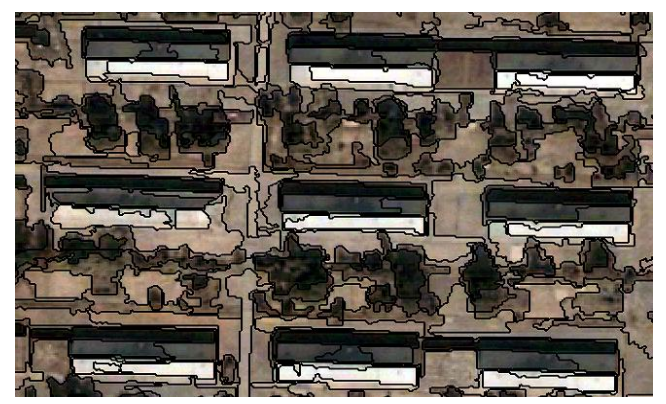

Figure 4. Subset of segmented image

\subsection{Feature Extraction}

As mentioned earlier, stable features are defined and variable features are extracted in this stage. In this paper, considering building definition, characteristics such as regular geometric shape, strong edge, and neighborhood with shadow are considered as stable features that are almost implemented in all cases by some little changes.

In this image considering this fact that all buildings in the image are rectangular, the similarity of objects to rectangle is considered as one of the stable features. In order to extract strong edges, canny edge detection algorithm with appropriate threshold has been utilized that results in keeping the objects that belong to building class. In the next stage, shadow is extracted by the rules that presented by Nussbaum (figure 5). Direct neighborhood with shadow is considered as one of the stable features in this algorithm. This feature derives from inherent characteristic of building as a high phenomenon. Features presented by Nussbaum to detect shadow are as follows:

$\begin{array}{ll}\text { Features } & \text { Threshold } \\ \text { Ratio B4 } & >0.199 \\ \text { Relative Border to brighter neighbors } & =1\end{array}$

Table 1. Classification rules of shadow class presented by Nussbaum

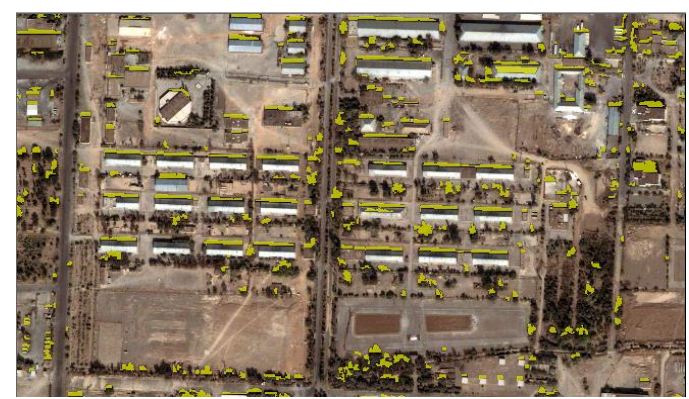

Figure 5. Detected shadows
Further, variable features that lead to optimal building detection are extracted by SEparability and THresholds tool. The feature analyzing tool SEaTH identifies characteristic features with a statistical approach based on training objects. These training objects represent a small subset out of the total amount of image objects and should be representative objects for each object class. The statistical measure for determining the representative features for each object class is the pairwise separability of the object classes among each other. Subsequently, SEaTH calculates the thresholds which allow the maximum separability in the chosen features (Nussbaum et al., 2008). Here, choosing training samples of building class and other classes in the image such as vegetation, road, wasteland, etc, features that result in optimal separation of probability distribution function between building class and other classes (two by two) are determined as optimal features with appropriate threshold.

Extracted variable features from this image have been presented in the following table:

\begin{tabular}{ll} 
Features & Threshold \\
\hline Ratio B2 & $>0.338$ \\
Ratio B4 & $<0.229$ \\
Mean NDVI & $<0.019$ \\
Length & $<80$ \\
Rectangular Fit & $>0.8$ \\
GLCM Homogeneity & $>0.07$ \\
Area & $<1200$
\end{tabular}

Table 2. Extracted variable features in this case study

Objects qualifying all the above conditions are identified as building based on extracted features (stable and variable).

\section{EVALUATION AND DISCUSSION}

As mentioned before, algorithm presented in this paper as a general algorithm can be implemented on high resolution satellite imagery in different areas. Central core of this algorithm includes stable and variable features. In this section, stable features have been derived from inherent characteristic of building. Variable features are also extracted by an analysis tool for optimum separation of building class from other classes in the studied image. Using stable and variable features together in a central core provide the possibility to generalize this algorithm. It is worth mentioned that changing the threshold value of stable features for extraction of strong edges and/or proximity degree of pre-defined geometric shape and even any shape other than rectangle prevents the user from making no changes in these features. Of course, this problem seems to will limit complete transferability of classification model to other data sets.

It is to be noted that determining Scale Parameter for segmentation in a trial and error process according to importance of the produced objects in all the next stages, giving a solution to obtain the scale parameter in an automatic process has a significant effect on algorithm automation process.

Though, in this algorithm, it has been tried to extract all buildings in the image by considering stable and variable 
features together, it could not be accomplished as shown in the below image (figure 6).

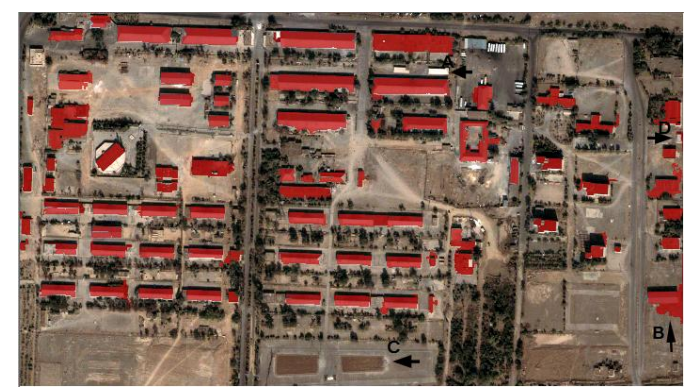

Figure 6. Resulted image

Extraction of over $80 \%$ buildings shown in the resulted image indicates successful applicability of algorithm presented in this research. Though, as mentioned, it was not successful in some cases, e.g. detecting buildings with light roofs (A). Since threshold of spectral features (Ratio B2 \& Ratio B4) are arranged for detection of dark roofs and gable roof, this conclusion seems reasonable. This is because of failing analysis tool in extracting suitable features which result in optimal separation of building class with light roof from other classes.

Also, in some cases, roof was not totally included in building class (especially in relation with middle line of buildings with gable roof). In these cases, objects with total vicinity with building class were regarded as building (Post-processing stage).

As shown in the above image, some objects are classified in building class by mistake. In these cases, the intended object includes part of building and part of the adjacent phenomenon (B) that algorithm allocates these objects to the building class entirely. In these cases, it is possible to consider a smaller scale parameter that leads to the production of smaller objects. Of course, quality of detecting other buildings was reduced upon such segmentation. In other cases where big objects were allocated to building class by mistake (for example, two dark rectangles on the bottom of the image (C)), these objects were omitted from building class using area feature. Also, in some cases, though image objects were small and building roof and adjacent phenomenon were not simultaneously covered by that object, it was inevitably included in building class (D) since all conditions were qualified

\section{CONCLUSION}

This paper tends to extract building using an object based image analysis approach. This method allows us to use neighborhood, contextual and geometrical features in addition to spectral features. It has been attempted to present a general algorithm for building extraction from different satellite image. In the first step, the image pixels from the image are grouped to form objects with the aid of multiresolution segmentation algorithm. Then the features that lead to robust building extraction will be determined. Features are divided into two classes in this algorithm: stable and variable. Stable features are derived from inherent characteristics of building phenomenon and they provide us the possibility to be implemented on different satellite images. Variable features, depending on the case, are extracted using a feature analysis tool (SEaTH). Implementing this algorithm on a part of Isfahan QuickBird imagery was successful in extracting over $80 \%$ existing buildings. Though in some cases the presented algorithm was not successful, the results were generally promising and the authors intend to examine complete transferability of classification model to other data sets and determine how much threshold values and features require adjustment and also find a solution for obtaining optimized scale parameter in an automatic process in the subsequent researches.

\section{REFERENCES}

Aminipouri, M., Sliuzas, R. and Kuffer, M., 2009. ObjectOriented Analysis of Very High Resolution Orthophotos for Estimating the Populations of Slum Areas, Case of Dar-EsSalaam, Tanzania, High-Resolution Earth Imaging for Geospatial Information, ISPRS Workshop, Hannover, Germany.

Benz, U.C., Hofmann, P., Willhauck, G., Lingenfelder, I. and Heynen, M., 2004. Multi-Resolution, Object-Oriented Fuzzy Analysis of Remote Sensing Data for GIS-Ready Information. ISPRS Journal of Photogrammetry and Remote Sensing, 58(34): 239-258.

Blaschke, T., 2010. Object based image analysis for remote sensing. ISPRS Journal of Photogrammetry and Remote Sensing. Vol. 65 (2010) 2_16.

Dutta, D. and Serker, N.H.M.K., 2005. Urban Building Inventory Development using Very High Resolution Remote Sensing Data for Urban Risk Analysis. International Journal of Geoinformatics, , 1(1).

Hofmann, P. (2001) Detecting urban features from IKONOS data using an object-oriented approach. First Annual Conference of the Remote Sensing \& Photogrammetry Society. Munich, Germany.

Hofmann, P., Strobl, J., Blaschke, T. and Kux, H., 2008. Detecting Informal Settlements from QuickBird Data in Rio de Janeiro Using an Object-Based Approach. In: T. Blaschke, S. Lang and G.J. Hay (Editors), Object-Based Image Analysis. Springer, Berlin Heidelberg, pp. 531-553

Nussbaum, S., and Menz, G., 2008. Object-Based Image Analysis and Treaty Verification. Springer Science+Business Media B.V. 